\title{
Complutum
}

ISSN: 1131-6993

\section{El plan arqueológico director de la cuenca del Navia. Balance de tres lustros de actividad arqueológica continuada en el Occidente de Asturias}

Ángel Villa Valdés ${ }^{1}$

Recibido: 05/04/2021 / Aceptado: 09/08/2021

Resumen. El Plan Arqueológico Director de la Cuenca del Navia $^{2}$ es el documento por el que el Principado de Asturias ordenó entre 1997 y 2009 las intervenciones sobre el patrimonio arqueológico de los territorios del Navia-Eo, en el extremo occidental de la región. Hasta entonces, a pesar del esfuerzo económico realizado por el Gobierno Regional, los resultados no fueron en estas comarcas demasiado satisfactorios en materia de conservación del patrimonio histórico por la carencia de un proyecto que definiese los objetivos y un modelo de gestión adecuado. Por esta razón se encargó un plan director que estableciese las prioridades de intervención arqueológica en sus diversas facetas: conservación, investigación y difusión. En la presente comunicación se dará cuenta de los criterios empleados en la selección de objetivos, del tipo de intervenciones propuestas y de las vías de financiación utilizadas. Asimismo se expondrán los avances producidos mientras el plan se mantuvo vigente y las causas que motivaron su abrupta interrupción.

Palabras Clave: Conservación, Investigación, Medio rural, Desarrollo, Turismo

[en] The Archaeological Plan Director of the Navia Basin. Balance of three decades of continued archaeological activity in western Asturias

Abstract. The Navia Basin Archaeological Master Plan is the document by which the Government of Principado de Asturias ordered between 1997 and 2009 interventions on the archaeological heritage of the Navia-Eo territories, in the extreme west of the region. Until then, despite the economic effort made by the Regional Government, the results in these regions were not very satisfactory in terms of conservation of historical heritage due to the lack of a project that would define the objectives and an adequate management model. For this reason, a master plan was commissioned to establish the priorities for archaeological intervention in its various facets: conservation, research and dissemination. This -paper will show the criteria used in the selection of objectives, the type of proposed interventions and the financing channels used. Likewise, it will present the progress made while the plan was in force and the causes that led to its abrupt interruption.

Keywords: Conservation, Research, Rural areas, Development, Tourism

Sumario. 1. Introducción. 2. Antecedentes: Asturias y el Programa Nacional de Parques Arqueológicos. 3. El valle del Navia como escenario arqueológico. 4. Un plan arqueológico para el Occidente de Asturias. 5. Planifica que algo queda. Referencias.

Como citar: Villa Valdés, Á. (2021): El plan arqueológico director de la cuenca del Navia. Balance de tres lustros de actividad arqueológica continuada en el Occidente de Asturias. Complutum, 32(2): 387-399.

\section{Introducción}

El valle del río Navia es una de las comarcas más singulares en el abigarrado mosaico de identidades paisajísticas y culturales que componen el Principado de Asturias. La personalidad de su relieve, arquitectura popular y lengua conforman una geografía con características genuinas, raramente conservadas en una región cuyas estructuras tradicionales se

\footnotetext{
Museo Arqueológico de Asturias angel.villavaldes@asturias.org

2 Esta comunicación se elaboró como parte del proyecto LOKI de I+D+i PID2019-104297GB-I00 financiado por MCIN/ y dirigido por la Dra. Inés Sastre Prats (IH-CSIC). AEI/10.13039/501100011033/
} 
han visto radicalmente transformadas en las últimas décadas.

Como consecuencia de su secular abandono, al que se suma la tardía y moderada mecanización de las labores agrícolas, una demografía débil, en imparable caída, y la arcaica red de comunicaciones que vertebran la comarca, los territorios del Navia atesoran aspectos de la sociedad rural asturiana extintos hace décadas en otras zonas de la región.

El resultado ha sido una cierta fosilización del paisaje tradicional de la que el patrimonio cultural y, en particular, el patrimonio arqueológico resultaron directos beneficiarios pues el grado de alteración de una parte sustancial de los bienes conocidos era, por lo general, limitado. Un atractivo poderosamente incentivado por la presencia de yacimientos célebres, algunos como el Castro de Coaña, de referencia inexcusable en la bibliografía especializada, o por la noticia de hallazgos en túmulos, cuevas o castros, muchos de ellos objeto de aventuras de sesgo arqueológico con resultados no siempre afortunados.

Con la excepción del Castro de Coaña (figura 1), a finales del pasado siglo, la mayor parte de los yacimientos explorados arqueológicamente se encontraban en un estado de conservación preocupante pues carecían de cualquier programa de control y mantenimiento. Un problema que previsiblemente se vería agravado por la progresiva introducción de maquinaria pesada en las tareas del campo y la implantación de nuevas actividades con fuerte impacto paisajístico como los parques eólicos o el desarrollo incipiente de una nueva forma de turismo, el rural, entregado a la búsqueda de recursos de cualquier orden con los que estimular una oferta de incierta demanda.

En estas circunstancias, el Gobierno del Principado de Asturias se planteó la necesidad de ordenar adecuadamente la actividad arqueológica para atajar con sus propuestas los riesgos previsibles de esta nueva situación, orientar sobre el uso más conveniente del patrimonio arqueológico y favorecer su explotación prudente como recurso cultural. El documento elaborado a tal fin se denominó Plan Arqueológico Director de la Cuenca del Navia y fue aprobado por el Pleno de la Comisión de Patrimonio Histórico de Asturias en su sesión de 19 de noviembre de 1997. En conjunto, un proyecto que afectaba a 17 municipios distribuidos sobre una superficie total de unos $1.600 \mathrm{Km} 2$.

Durante la década en que el Plan Director se mantuvo vigente, con un lustro epigonal de actividad discontinua, más de una veintena de conjuntos arqueológicos fueron objeto de intervención. Campos tumulares y megalitos, estaciones con arte rupestre, poblados fortificados de la Edad del Hierro y explotaciones auríferas de época romana motivaron iniciati-

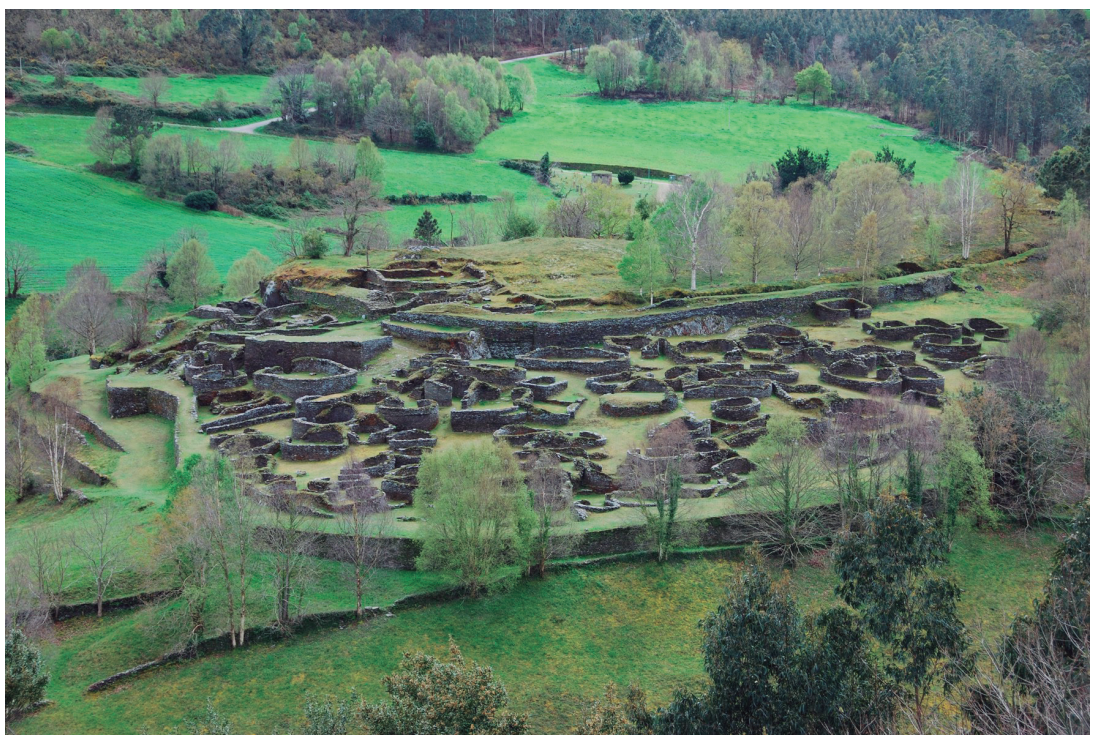

Figura 1. Castro de Coaña. Primera opción del Gobierno Regional para la creación de un parque arqueológico que resultó finalmente descartado por el Ministerio de Cultura en favor del castro de la Campa Torres, en Gijón. 
vas de muy diversa entidad en las que, junto con trabajos de mantenimiento, desbroce o jardinería, también se realizaron excavaciones si bien vinculadas, por lo general, a proyectos de conservación y musealización de lugares arqueológicos. La visibilidad que este conjunto de yacimientos adquirió en muy poco tiempo animó a su adaptación como guion del programa supramunicipal de desarrollo denominado Parque Histórico del Navia en cuyo órgano de gestión, sin embargo, no se contempló la participación de ningún experto en materia de patrimonio, siendo gestionado de facto por los alcaldes y alcaldesas de los ayuntamientos implicados.

En este texto se recordarán los criterios empleados en la selección de objetivos, el tipo de iniciativas propuestas y los agentes participantes en cada una de ellas pero añadiendo, con la perspectiva que proporcionan los años transcurridos desde su aprobación, una valoración ponderada de lo conseguido tras un periodo inédito de actividad patrimonial planificada en el marco del denominado Plan Arqueológico del Navia-Eo.

\section{Antecedentes: Asturias y el Programa Nacional de Parques Arqueológicos}

En 1987, el Instituto Central de Conservación y Restauración de Bienes Culturales seleccionó el castro de la Campa Torres para su Plan Experimental de Parques Arqueológicos. Las excavaciones en el yacimiento, identificado por José Manuel González como la Noega de las fuentes clásicas y para el cual los profesores universitarios José Luis Maya y Miguel Ángel de Blas habían tramitado una primera solicitud de financiación en 1977, dieron comienzo finalmente en 1978 codirigidos por el primero de ellos y Juana Bellón. Fue aquella intervención el embrión del Proyecto Gijón de Arqueología que, a partir de 1981, habría de impulsar Manuel Fernández Miranda, primero en su condición de Subdirector General de Arqueología, luego como Director de Bellas Artes del Ministerio de Cultura, programa clave para el extraordinario desarrollo de la actividad arqueológica que desde entonces promueve la ciudad de Gijón y los importantes recursos patrimoniales con ella generados (Fernández Ochoa, 1996).

La elección de la Campa Torres se produjo en respuesta a la consulta formulada desde el Ministerio a las Comunidades Autónomas para la selección de "yacimientos preferentes” (Querol, 1993: 15). Una decisión que en Asturias motivó un fuerte debate entre administraciones pues, frente a la opción ganadora, reivindicada por un ayuntamiento con enorme peso político y respaldada desde el Ministerio de Cultura por el gijonés Fernández Miranda, el novísimo gobierno regional era partidario de crear su primer parque arqueológico en el occidente de Asturias, tomando el Castro de Coaña como elemento central de su discurso. Una intención a la que probablemente no fue ajena la inequívoca voluntad, en aquellos primeros años de Autonomía, de ofrecer alternativas al que ya se vislumbraba como incierto futuro de las comarcas de la Asturias occidental. Un tiempo en el que se impulsó, junto con la mejora de prestaciones sociales y sanitarias esenciales, el fomento de nuevos destinos turísticos singulares en espacios ultraperiféricos que se sustentaban en recursos de orden ambiental y cultural. Fue el caso del hotel La Rectoral, en Taramundi, o el definitivo respaldo institucional al proyecto personal de José María Naveiras, Pepe el Ferreiro, y su Museo Etnográfico de Grandas de Salime. En esa misma línea cabe interpretar el intento fallido del Ministerio de Cultura para crear un Parque Etnográfico en Somiedo, con el paisaje tradicional de brañas y ganadería extensiva como motivo central (López y Graña, 1993).

Fue en este contexto en el que el Gobierno Regional manifestó la voluntad de mantener su propuesta y afrontar, en paralelo a la acción del Ministerio, la creación de un parque propio en Coaña. Con esta intención, a partir de 1987 la Consejería de Cultura promovió importantes inversiones en el yacimiento destinadas a sufragar la investigación arqueológica, la ejecución de obras de consolidación de las ruinas y la creación de infraestructuras de acogida para visitantes e investigadores.

Por desgracia, buena parte del empeño puesto por el Principado de Asturias en mejorar el aspecto general del yacimiento y sus condiciones de visita se disolvió en sofisticados proyectos de intervención, con alto coste pero de ejecución inadecuada y aportaciones anecdóticas al conocimiento arqueológico del asentamiento (Carrocera, 2003; Villa, 2010 y 2019). Del mismo modo, las modernas instalaciones que incluían aparcamiento, dos edificios con aula didáctica, almacén, sala de proyecciones y cafetería, de acuerdo con el 
proyecto de los arquitectos Mariano Sánchez y Félix Gordillo, aunque fueron inauguradas oficialmente en noviembre de 1993, carentes de un plan de gestión, nunca llegaron a operar satisfactoriamente (figura 2). Por su parte, el proyecto de parque arqueológico no llegó a ser ni tan siquiera esbozado.

\section{El valle del Navia como escenario arqueológico}

Más arriba se han apuntado las razones de orden político que justificaban el empeño asturiano por impulsar la creación del parque arqueológico en Coaña pero esta intención oficial no era caprichosa pues estaba respaldada por la existencia de un conjunto patrimonial poco común, con ruinas monumentales y un potencial científico todavía por explorar.

La actividad arqueológica en el valle del río Navia, con los castros como motivo principal de la investigación, contaba con un largo historial de intervenciones que se remontan a los propios orígenes de la Arqueología como disciplina científica.

En enero de 1877, José María Flórez y González, un maestro natural de Cangas del Narcea y miembro de la Sociedad Económica de Amigos del País, obtuvo el patrocinio de la Comisión Provincial de Monumentos para la realización de excavaciones arqueológicas en Coaña, lugar que había conocido en julio de 1876. Los postulados metodológicos que animaron la labor de Flórez y la cuidada publicación de resultados podrían figurar hoy, de haber desarrollado sus investigaciones en yacimientos británicos o franceses, como referencias pioneras en la práctica científica de la Arqueología (Flórez, 1878; Villa, 2010: 142). Fue no obstante Antonio García y Bellido quien, con las investigaciones realizadas, junto con Juan Uría, a partir de 1940 en este yacimiento y en el vecino castro de Pendia, quien afianzó, con sus publicaciones y magistrales dibujos, los poblados fortificados del Navia como referencia genuina en el acervo científico de la arqueología peninsular (García y Bellido, 1941 y 1942; García y Bellido y Uría, 1942; Fernández Ochoa \& Villa, 2004; Uría y García y Bellido, 1943).

A partir de entonces, las razones que animaron el interés de García y Bellido por Coaña, justificadas en el contexto europeo de la época especialmente atento a la definición del mapa étnico y cultural de los pueblos mediante el estudio de la cultura material (García-Bellido, 2002: 42) continuaron alentando un número considerable de intervenciones arqueológicas y consiguiente incorporación de nuevos yacimientos a la lista de ruinas arqueológicas, en principio, susceptibles de visita.

Lo cierto es que, a pesar del esfuerzo presupuestario, del incremento de lugares excavados y la creación de instalaciones como las de Coaña, a mediados de los años noventa los

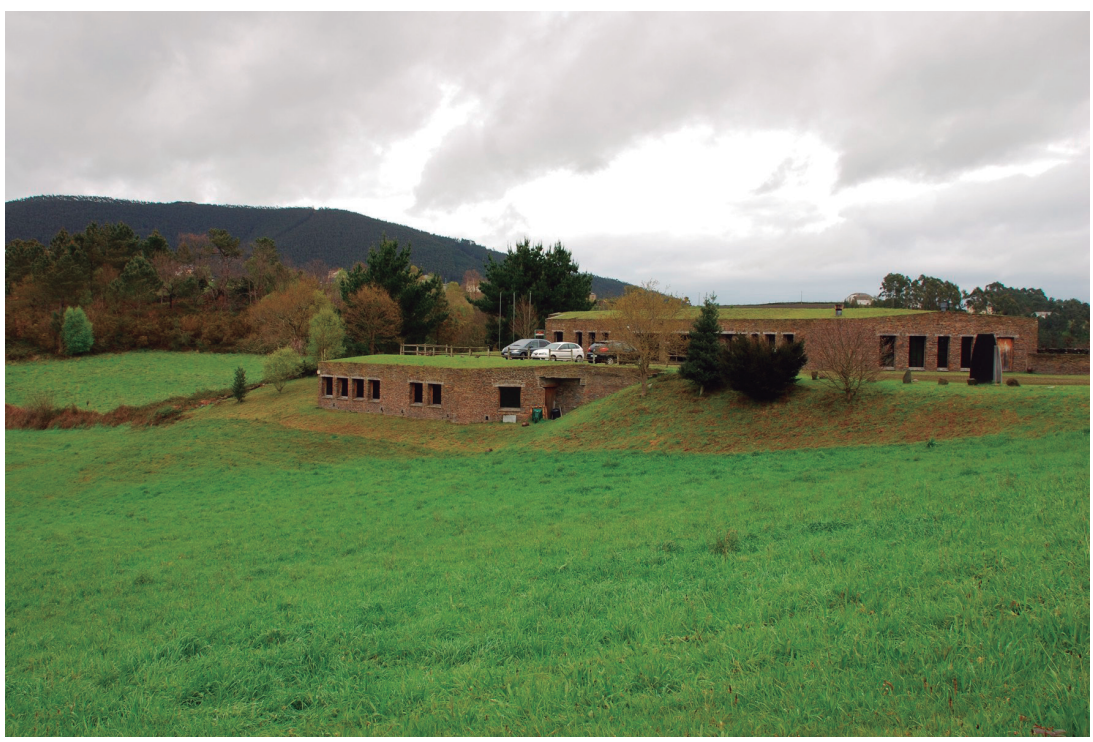

Figura 2. Castro de Coaña. Aparcamiento e instalaciones de acogida de visitantes con aula didáctica y oficina de información. Equipamientos inaugurados en 1993. 
resultados no eran satisfactorios. En ausencia de una mínima planificación, la situación derivó en un desarrollo desordenado de las actuaciones, fundamentalmente excavaciones arqueológicas, que no redundaron en la mejora del estado de conservación de las ruinas ni en su mejor acondicionamiento para la visita. El desinterés de la mayor parte de municipios agravó este proceso que culminó en no pocas ocasiones con el total abandono de los restos arqueológicos precisamente en el momento en que eran potenciados como singular reclamo en la oferta turística regional y poderoso elemento identitario.

Con el fin de corregir estas contradicciones la Consejería de Cultura encargó en 1997 la redacción de un plan director que estableciese las prioridades de intervención arqueológica en el occidente de Asturias y el ámbito territorial específico en el que debería aplicarse (Vi1la, 1999: 205).

\section{Un plan arqueológico para el Occidente de Asturias}

Este documento fue el Plan Arqueológico Director de la Cuenca del Navia. Una denominación inexacta pues, en realidad, el área de actuación comprendía los diecisiete concejos del interfluvio Navia-Eo, un espacio geográfico y cultural con marcados rasgos diferenciales respecto al resto de Asturias, vigentes, en parte, como consecuencia del atraso secular sufrido por estas comarcas y que, por ejemplo, moderó los catastróficos efectos que la mecanización intensiva del medio rural había ocasionado sobre el patrimonio arqueológico en otros lugares.

En la selección de objetivos se consideraron aspectos tales como la fragilidad de las ruinas, su expresividad o la sostenibilidad de las intervenciones a desarrollar, cuestión en la que el compromiso de colaboración de las entidades locales fue considerado factor determinante. Se aplicaban, en definitiva, los criterios de la Carta de Turismo Cultural adoptada por ICOMOS (Consejo Internacional de Monumentos y Sitios) en 1976 y manejados como elementos diagnósticos para la puesta en marcha de los primeros parques arqueológicos en España (Querol, 1993: 16). De manera resumida, se consideraron cuestiones jurídicas relacionadas con la propiedad y rango de protección, el estado de conservación y monumentalidad de los restos arqueológicos como punto de apoyo clave para la comprensión del yacimiento y de sus componentes principales, el interés científico, educativo o histórico, la calidad paisajística y agentes molestos en el entorno del yacimiento así como los riesgos o efectos negativos que la promoción turística pudiera originar sobre el mismo y su medio (figura 3 ).

El tipo de intervención propuesta en cada lugar, aun considerando la representatividad o la relevancia científica de cada elemento, fue determinada en función de criterios prácticos, de oportunidad o urgencia, procurando adaptar a cada caso y con las limitaciones inevitables, las recomendaciones internacionales en materia de conservación y promoción de yacimientos arqueológicos. No se primaron las excavaciones arqueológicas. Conscientes de que la comprensión y disfrute de un yacimiento es posible mediante otros recursos, especialmente cuando éste y su entorno ofrecen la expresividad que caracteriza el patrimonio aquí considerado, los trabajos de excavación fueron restringidos, de acuerdo con las directrices elaboradas por el Centro Internacional de Estudios para la Conservación y la Restauración de los Bienes Culturales (ICCROM 1986: 9), a propuestas donde se ofrecieran garantías de estudio, consolidación y publicación de resultados.

De esta forma, un grupo de veinticinco yacimientos fueron seleccionados como objetivos del Plan Director entre los que se contaban monumentos tumulares y megalíticos, dos estaciones con arte rupestre, varios castros y diversas cortas e infraestructuras auxiliares relacionadas con el beneficio aurífero en época romana. En los años de vigencia del Plan Director, desarrollado en programas anuales como Plan Arqueológico del Navia-Eo, nuevos yacimientos se incorporaron al mismo mientras que, excepcionalmente, algún otro resultó excluido.

Las actuaciones de carácter general comprendían intervenciones básicas de limpieza y desbroce, el diseño y ejecución de un programa de señalización para crear o, en su caso, normalizar, la diversidad de formatos y contenidos ya existentes y la edición de material divulgativo. Éstas se complementaban con propuestas específicas para determinados yacimientos destinadas a subsanar deficiencias documentales (esencialmente planimetrías), a promover modos de colaboración estable con los centros escolares, a mejorar los servicios 


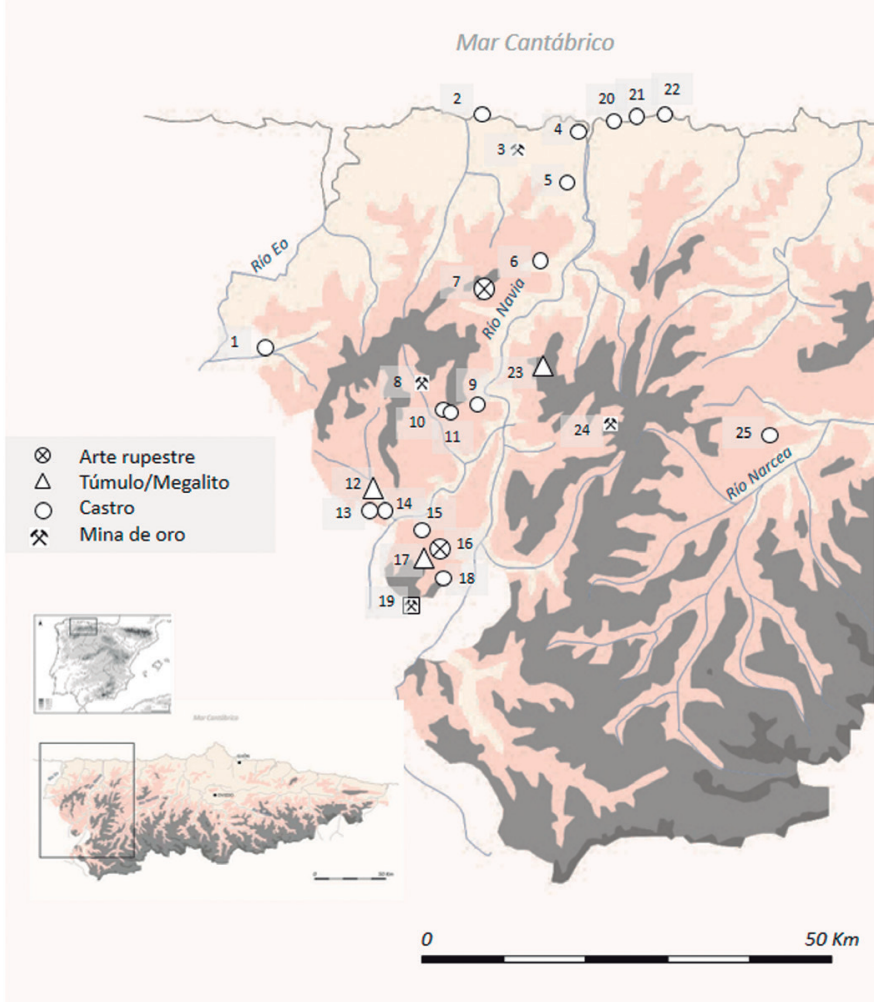

Figura 3. Área comprendida en el Plan Arqueológico del Navia-Eo con indicación de los lugares arqueológicos donde se realizó algún tipo de intervención: 1. Os Castros, Taramundi;

2. Cabo Blanco, 3. Andía; 4. Mohías; 5. Coaña; 6. Pendia; 7. Cova del Demo; 8. Arruñada;

9. El Castelón de Illano; 10. Pico da Mina; 11. San Isidro; 12. Brañavella; 13. Cortín dos Mouros;

14. Os Castros de Ferreira; 15. Chao Samartín; 16. A Xorenga; 17. Canadeiro; 18. Monte Castrelo de Pelóu; 19. Penafurada; 20. El Castrillón de Téifaros; 21. El Castiel de Soirana;

22. El Castro de Vigo; 23. Pedar da Filadoira; 24. A Freita; 25. San Chuis.

de guardería e información turística, a promover la creación de instalaciones museísticas, a incentivar la investigación arqueológica $\mathrm{y}$, fundamentalmente, a promover intervenciones periódicas de conservación y consolidación de yacimientos (figura 4). En la práctica, las intervenciones fueron tan diversas como las circunstancias concurrentes en cada caso si bien procurando la colaboración institucional entre el Principado de Asturias, los ayuntamientos y la propiedad privada cuando así se requería.

Las partidas presupuestarias dispuestas anualmente por el Principado de Asturias eran complementadas con las subvenciones del Instituto Nacional de Empleo, sustituido tras la transferencia de competencias por el Servicio de Empleo regional, las aportaciones puntuales y finalistas de cada ayuntamiento en aquellas actuaciones que les resultasen de interés o con fondos de programas europeos vincula- dos con la protección del patrimonio cultural. Asociaciones privadas, como la Asociación de Amigos del Parque Histórico o la Asociación de Hostelería de Taramundi (Menéndez y Villa, 2009; Villa, 2007; Villa et al., 2007) y, ocasionalmente, alguna empresa privada asumieron de manera excepcional la financiación de gastos y contrataciones en algunos ejercicios. En todo caso, fuese cual fuese el marco administrativo y los agentes implicados, 1o destacable es que los criterios de actuación y la dirección técnica de los trabajos contaron con la participación de un equipo estable, aunque inevitablemente sometido a la estacionalidad de los contratos, y en todo momento, con independencia de la entidad contratante, coordinado desde el Servicio de Patrimonio Histórico de la Consejería de Cultura del Principado de Asturias, desde donde se ejerció la dirección del Plan durante los años de vigencia. 


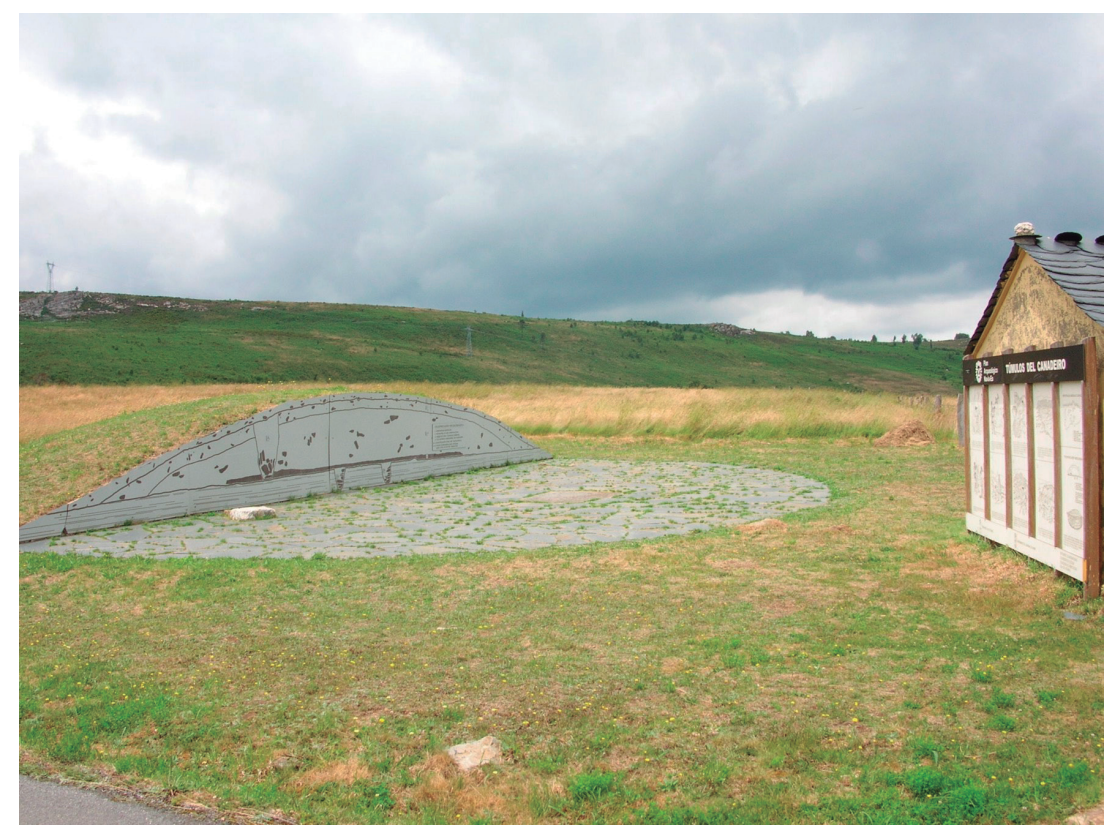

Figura 4. Túmulo neolítico de El Canadeiro, Xestoselo (Grandas de Salime). Intervención de rescate sobre una estructura parcialmente destruida.

En la imagen se muestra su aspecto tras el acondicionamiento para su vista en espacio abierto y sin custodia.

El Plan Arqueológico se fue consolidando así como proyecto supramunicipal, abierto y acumulativo, en el que, aplicando como norma principios de subsidiariedad y sostenibilidad, pronto pudieron advertirse los primeros rendimientos tanto en el ámbito económico como, científico y social.

Más allá de los ingresos generados entre la población local como consecuencia de las periódicas convocatorias de empleo, la creciente participación de estudiantes y colaboradores universitarios, la realización de estudios complementarios, el interés creciente despertado por las informaciones aparecidas en los medios de comunicación y la implicación, más allá de sus obligaciones contractuales, del personal técnico en proyectos de investigación vinculados con las actividades del Plan favorecieron la percepción de continuidad de la actividad arqueológica en un medio social en el que la figura de los profesionales de la arqueología y de la conservación perdió su condición de acontecimiento exótico para ser percibida como agente útil en el desarrollo local, en la proyección pública de los recursos propios $\mathrm{y}$, en definitiva, como estímulo para la autoestima colectiva.

La respuesta social fue, por tanto, muy positiva y la mayor parte de ayuntamientos to- maron como propios los objetivos propuestos en el Plan Director. Un año después, en 1998, una vez constituido el Centro de Desarrollo Navia-Porcía que habría de gestionar los fondos asignados a la comarca en el programa PRODER (Programa de Desarrollo Rural), los compromisos se concretaron con nueve de los municipios en un plan inversiones que asumía íntegramente los objetivos y estrategias del Plan y que habría de desarrollarse en el marco de una figura promocional de nueva creación, el Parque Histórico del Navia, en el que se integraban los concejos de Boal, Coaña, El Franco, Grandas de Salime, Illano, Navia, Pesoz, Tapia de Casariego y Villayón. Un proyecto comarcal definido como producto cultural y turístico con el que se aspiraba "a conformar un espacio rural sostenible basado en el mejor aprovechamiento de todos los recursos endógenos existentes".

El Parque Histórico del Navia podía interpretarse, en principio, como un gran avance pues significaba que un conjunto amplio de municipios, con importantes carencias asistenciales, con déficit endémico de infraestructuras básicas, decidía sin embargo tomar como idea motriz para su desarrollo comarcal el reconocimiento y valorización de su patrimonio cultural. De hecho, una de las primeras deci- 
siones consensuada por sus representantes fue la creación, en el entorno del castro de Chao Samartín, en Grandas de Salime, de un centro museístico que habría de centralizar las actividades relacionadas con el tratamiento y custodia de los materiales arqueológicos recuperados en las diversas intervenciones y favorecer el trabajo del extenso grupo de profesionales e investigadores nacionales y extranjeros que con regularidad colaboraban en las tareas de estudio y restauración (figura 5).

El proyecto del edificio fue encargado por la Consejería de Cultura del Principado de Asturias a los arquitectos Joaquín Menéndez y Pablo Gamonal quienes, en colaboración con el equipo arqueológico, elaboraron un documento en el que se planteaba la construcción de instalaciones suficientes para albergar, además de una sala de exposiciones y otros servicios de atención al visitante, almacén y laboratorio. La obra fue ejecutada con cargo a los presupuestos comunes del Programa PRODER e inaugurada en abril de 2007 (García y Gamonal, 2009).

El centro, aunque promocionado con la denominación Museo Castro de Chao Samartín no llegó a ver formalizada tal condición administrativa por razones de diversa índole. Desde el principio fue concebido como centro subordinado al Museo Arqueológico de Asturias. El yacimiento era ya reconocido como lugar relevante en el campo de la arqueología protohistórica peninsular. Contaba, también, con una trayectoria consolidada como recurso arqueo- lógico visitable en el que se ofrecía servicio permanente de guías en varias lenguas. Ambas circunstancias, reconocimiento científico y potencial turístico, fueron consideradas claves en la redacción del proyecto museológico y su consiguiente implantación museográfica (Hevia, 2009: 61). Con el objetivo de procurar su autosuficiencia, el edificio se presentaba como uno de los centros de cabecera o "puerta" para la recepción de visitantes del Parque Histórico del Navia pero además, y sobre todo, como centro de trabajo en el que el variado repertorio arqueológico producido en el marco del Plan Arqueológico Director de la Cuenca del Navia alimentase el interés de instituciones científicas y académicas para colaborar con el Museo así como en las actividades a realizar en el Chao Samartín y otros yacimientos de la comarca (Villa, 2009). En el proyecto, el nuevo edificio y el yacimiento se presentaban como partes inseparables de un todo (figura 6): el museo, concebido esencialmente como "un lugar de trabajo arqueológico en el que se procura, con éxito, relacionar los procedimientos científicos que han permitido reconstruir la historia del lugar y su morfología con el yacimiento y la exposición permanente" (Franch, 2009).

Durante sus primeros años de andadura (2007-2009), el centro contó con una doble gestión, encomendada a la Fundación Parque Histórico del Navia y el Ayuntamiento en lo referente a la atención al visitante, mientras que los aspectos de orden patrimonial se man-

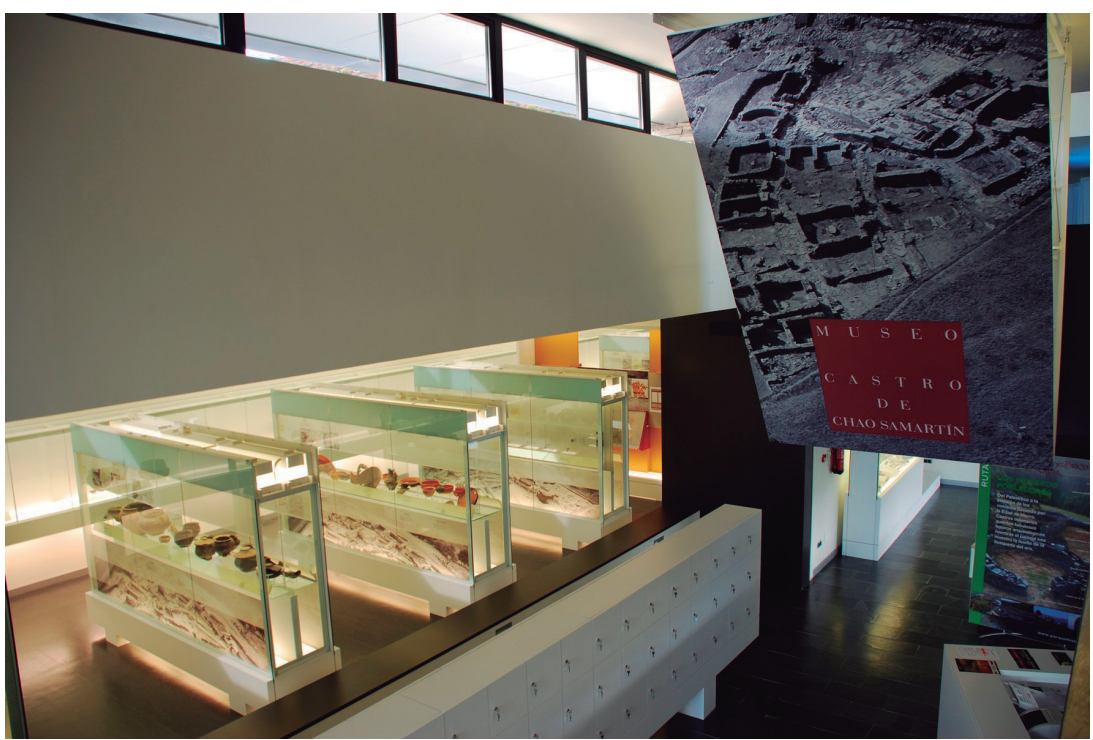

Figura 5. Vista interior del Museo Castro de Chao Samartín, en Grandas de Salime. Proyecto arquitectónico de Menéndez \& Gamonal inaugurado en abril de 2007. 


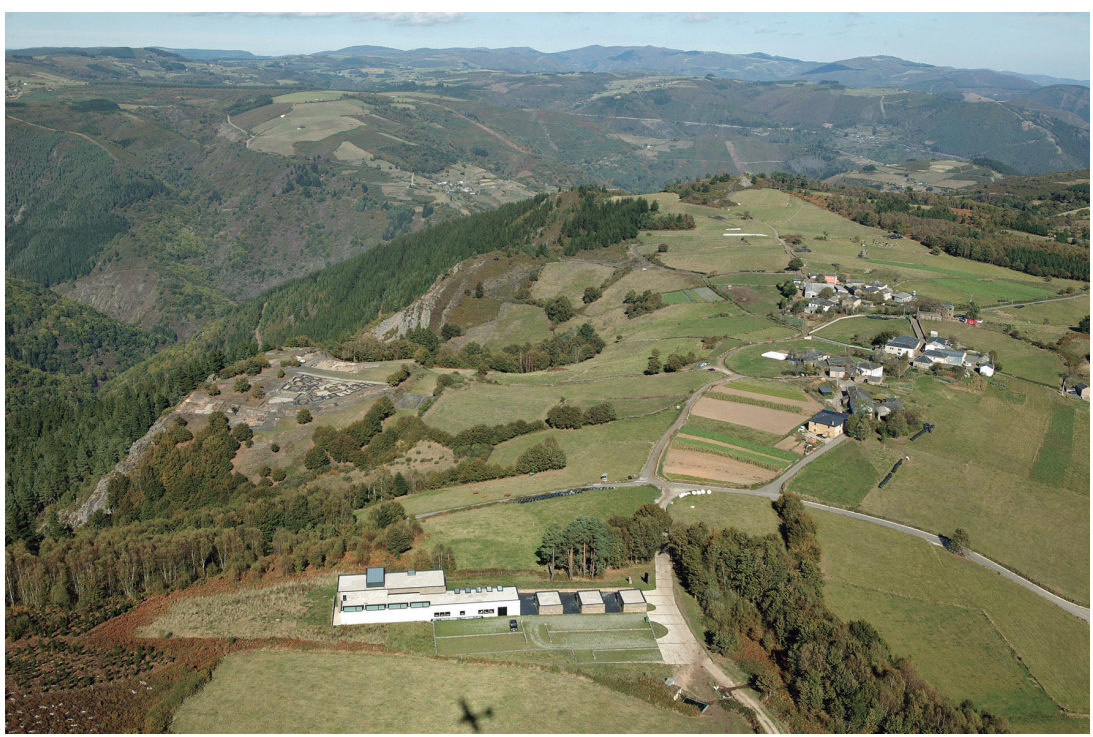

Figura 6. Vista panorámica de las instalaciones museísticas, en primer término,y el castro de Chao Samartín a la izquierda. A la derecha la población de Castro.

tuvieron bajo control exclusivo de la Consejería de Cultura del Principado de Asturias. Hasta 2009 la supervisión técnica de la colección arqueológica fue desempeñada por Susana Hevia González, arqueóloga con titulación específica en gestión cultural y museología. En este periodo se editó el catálogo íntegro de la colección permanente, se puso en marcha la página web del sitio y la visita guiada al yacimiento podía realizarse en castellano, inglés y francés. Además, como ya se ha dicho, el Museo del Chao Samartín pasó a ser destinatario de los hallazgos producidos en las excavaciones programadas en el territorio del Navia-Eo.

La dotación de las nuevas instalaciones reforzó el avance en las investigaciones arqueológicas patrocinadas por la Consejería de Cultura del Principado de Asturias. La continuidad en el apoyo institucional permitió verificar la extraordinaria riqueza e importancia histórica de yacimientos como el Chao Samartín, Taramundi o Cabo Blanco, hasta entonces inéditos, y recuperar para los castros asturianos la notoriedad de antaño en el ámbito de la Arqueología protohistórica española (figura 7). Son expresión de la vitalidad alcanzada por el proyecto la decena de trabajos de investigación y tesis doctorales, las decenas de comunicaciones debatidas en reuniones científicas y el centenar largo de títulos aparecidos en libros y revistas especializadas.

Constituida la Fundación que habría de gestionar Parque Histórico del Navia, pronto pudo comprobarse el inexistente interés por soste- ner los objetivos iniciales, reemplazados por prioridades ajenas al estudio y la promoción del patrimonio cultural. En realidad, su deriva conceptual no diverge de la advertida en la política autonómica durante los años finales de la primera década de siglo, en la que los principios que animaron la corrección de los desequilibrios históricos de los territorios occidentales fueron poco a poco diluyéndose para retornar, en muy poco tiempo, a estrategias localistas que relegaron, hasta su práctica extinción, la gestión común, metódica y científica de las iniciativas hasta entonces puestas en marcha.

Las consecuencias resultaron catastróficas para elementos destacadísimos del patrimonio arqueológico pero también para la confianza de quienes habían depositado enormes esperanzas en un modelo de desarrollo que se había revelado factible, socialmente útil y sostenible con recursos muy discretos. En ese mismo tiempo, con las primeras hostilidades políticas, pudo comprobarse de manera dramática su fragilidad cuando el centro y el yacimiento fueron cerrados a los investigadores e interrumpida la actividad arqueológica en todas sus facetas, también la de conservación de una sobresaliente colección arqueológica. Y todo ello ante la incomprensible indolencia de las autoridades competentes en materia de patrimonio cultural (Villa, 2014; Villa y Hevia, 2014).

Los intentos que a partir de 2011 realizaron diferentes equipos de gobierno desde la Consejería de Cultura para recuperar una cierta nor- 


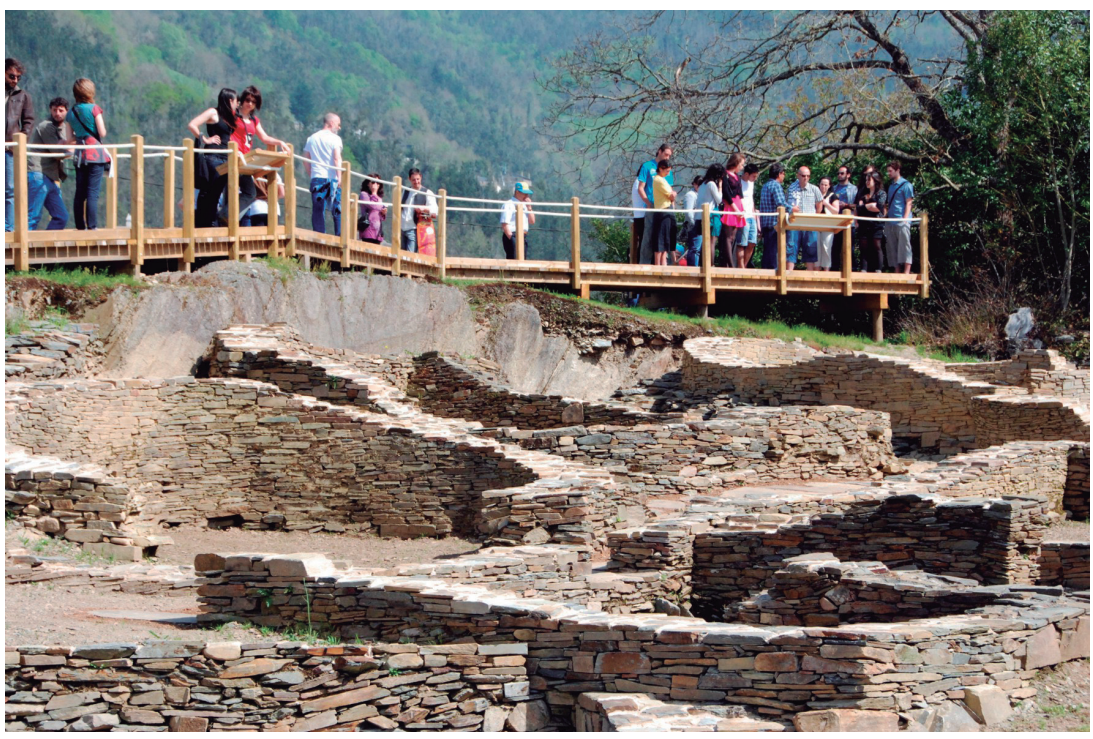

Figura 7. Os Castros de Taramundi. Su excavación se inició en el año 2000 como parte de las actividades programadas en el Plan Arqueológico del Navia-Eo. Los trabajos de consolidación y acondicionamiento para la visita concluyeron en 2010.

malidad en la gestión de aquel espacio arqueológico, yacimiento y colecciones, chocaron frontalmente con la incomprensible actitud de ciertas autoridades que, en el caso de Grandas de Salime, sembró la peor incertidumbre sobre el futuro, no sólo de sus recursos arqueológicos sino también de una institución cultural de referencia en Asturias, el Museo Etnográfico "Pepe el Ferreiro", principal atractivo turístico en la comarca. La situación se degradó hasta tal punto que el Principado de Asturias hubo de recurrir a sucesivos autos judiciales para poder afrontar trabajos urgentes de conservación y restauración en el castro de Chao Samartín (Montes y Villa, 2018; Villa et al. 2018; Villa, 2014).

Lamentablemente, en el momento en que se redacta este artículo, este mismo yacimiento es objeto de una intervención arquitectónica de promoción municipal que desatiende de manera flagrante los sucesivos dictámenes emitidos por ICOMOS España e ICOMOS Consejo Internacional de Itinerarios Culturales en los que se recomendaba la total refacción del documento por su impacto sobre las ruinas y por comprometer seriamente los valores que hacen de éste un lugar de referencia para la historia de Asturias .

\section{Planifica que algo queda}

Los años de trabajo continuado en el tercio occidental de Asturias a partir de la redacción del Plan Arqueológico Director de la Cuenca del Navia representaron una experiencia sin precedentes en nuestra región. Fueron la más positiva expresión de un tiempo en el que los criterios científicos prevalecían en la toma de decisiones y consiguiente orientación de la acción administrativa. La elaboración de los inventarios arqueológicos, el desarrollo de planes directores de ámbito supramunicipal, la puesta en marcha de proyectos editoriales (científicos y de divulgación) o la participación de los arqueólogos en los organismos de gestión ambiental fueron medidas adoptadas entonces con una repercusión inmediata sobre la protección de los yacimientos, que supusieron, además, un salto cualitativo en el conocimiento de nuestro patrimonio arqueológico.

En el ámbito de la arqueología urbana, en estos mismos años se desarrolló el denominado "Proyecto Gijón". Como resultado de un amplio programa de intervenciones y seguimiento sistemático de obras, el municipio pudo ofrecer en un corto plazo de tiempo una imagen renovada y sorprendente de la historia de la ciudad, especialmente de su periodo romano, y favoreció la feliz recuperación de restos arqueológicos y su conversión en destacados recursos patrimoniales (Fernández Ochoa, 1996 y 2005). Sin alcanzar los ejemplares resultados de proyectos como el Atlas del Subsuelo Arqueológico promovido por el Consejo de Europa para las ciudades belgas (Jurion-Dewaha, 
1999), en España el "Proyecto Gijón" contaba con la referencia de otras iniciativas con excelente balance como los protagonizados en Cataluña por el Taller Escuela de Arqueología de Tarragona (TED'A) (Ruiz de Arbulo y Mar, 1997) o, en Extremadura, el Consorcio de la Ciudad Monumental de Mérida junto con el Museo Nacional de Arte Romano y el Instituto de Arqueología de Mérida (Durán y Rodríguez, 2004). Todos ellos núcleos urbanos donde se procuró tratar la ciudad como yacimiento único, como espacio físico depositario de una secuencia histórica de larga duración, de impronta rastreada en un complejo mosaico de superficie finita e irremplazable y cuya reconstrucción fue posible por la adopción de medidas de sencilla praxis como la planificación de las intervenciones, la unificación de los sistemas de registro o la dirección coordinada de los trabajos.

Volviendo al caso que nos ocupa, al margen de los conflictos señalados, muy graves y que, ciertamente, revelan la debilidad creciente de la acción técnico-administrativa en materia de patrimonio cultural ante los vaivenes políticos, el balance que hoy ofrece el trabajo desarrollado de manera continuada entre 1998 y 2010 puede considerarse positivo. Lo es en términos operativos y sociales pues durante más de una década, con presupuestos muy limitados, la Consejería de Cultura no sólo mantuvo en condiciones adecuadas los yacimientos encomendados directamente a su gestión sino que propició la incorporación de nuevos e importantes recursos arqueológicos a la oferta cultural de Asturias, reorientando programas y vías de financiación ajenas que se canalizaron, bajo su coordinación, hacia la mejora de un importante conjunto de bienes patrimoniales, muchos de los cuales son hoy elementos muy destacados en la oferta cultural de sus municipios.

La continuidad de las actividades arqueológicas en el territorio del Navia-Eo y la dimensión científica exigida a cada intervención, con independencia de su orientación investigadora o meramente curativa, permitió explorar nuevos yacimientos y ampliar notablemente el conoci- miento de otros ya conocidos. En el ámbito de la arqueología castreña, los estudios desarrollados en el contexto de obras de consolidación o saneamientos han modificado de forma radical la comprensión del hábitat fortificado protohistórico y han favorecido la formación especializada de un amplio grupo de profesionales que trabajando conjuntamente en diferentes yacimientos han vinculado sus proyectos de investigación hacia el estudio de este territorio desde la Prehistoria hasta la Edad Media. Un valor añadido que el Principado de Asturias supo advertir al concebir la investigación arqueológica como fundamento primordial de la puesta en valor y promoción del patrimonio.

Es cierto que una parte de este trabajo se vio truncado de forma violenta cuando comenzaban a darse las condiciones para optimizar el rendimiento de equipos e instalaciones pero no lo es menos que la labor realizada ha otorgado visibilidad a una parte particularmente representativa de nuestro patrimonio. Las reuniones científicas organizadas en el marco del Plan Director, especialmente los Coloquios de Arqueología en la Cuenca del Navia, las exposiciones temporales, los ciclos de conferencias, la presentación de resultados en decenas de congresos internacionales, la renovación motivada por los nuevos descubrimientos en la exposición permanente del Museo Arqueológico de Asturias o los varios centenares de entradas bibliográficas generadas en estos años son logros reales, como también lo es la formación especializada de un amplio equipo de profesionales que, inevitablemente, al tiempo que profundizaban en el conocimiento de este territorio, han arraigado en mayor o menor medida en el tejido social que lo sustenta.

Por tanto, los ingredientes necesarios para que en algún momento pueda retomarse la planificación como herramienta clave de la acción patrimonial, para que la reflexión y el criterio científico vuelvan a primar frente a la arbitrariedad y el favor político, ya existen, sólo resta activarlos. Hay indicios que tal puede ser la voluntad de quienes ahora gobiernan. Crucemos los dedos.

\section{Referencias}

Durán Cabello, R. M. y Rodríguez Martín, F.G. (2004): Veinticinco años de arqueología urbana en Mérida, Cuadernos de Prehistoria y Arqueología de la Universidad Autónoma de Madrid 30, 153-166.

Fernández Ochoa, C. (1996): "Historia del Proyecto Gijón de Arqueología", en Complutum Extra 6 (I). Madrid, 29-37. 
Fernández Ochoa, C. (2005): «Investigación en la ciudad de Gijón desde una perspectiva arqueológica: luces y sombras de la arqueología urbana», El espacio urbano en la Europa Medieval: Nájera. Encuentros Internacionales del Medievo. Nájera, 61-76.

Fernández Ochoa, C. y Villa Valdés, A. (2004): "El castro de Coaña antes y después de García y Bellido: claroscuros en el tránsito de la erudición al discurso científico" en J. Blanquez Pérez y M. Pérez Ruiz (Ed. Cient.): Antonio García y Bellido. Miscelánea. Serie Varia 5. Madrid, 129-141.

Flórez y González, J. M. (1877): Memoria relativa a las excavaciones de El Castellón en el Concejo de Coaña (Asturias). Oviedo.

Franch, E. (2009): "El proyecto museográfico", en Á. Villa (Ed.): Museo Castro de Chao Samartín. Catálogo. Consejería de Cultura y Turismo del Principado de Asturias y Asociación de Amigos del Parque Histórico del Navia. Oviedo, 47-59.

García Menéndez, J. \& Gamonal Lombardero, P. (2009): "El proyecto arquitectónico", en Á. Villa (Ed.): Museo Castro de Chao Samartín. Catálogo. Consejería de Cultura y Turismo del Principado de Asturias y Asociación de Amigos del Parque Histórico del Navia. Oviedo, 41-45.

Hevia González, S. (2009): "El proyecto museológico", en Á. Villa (Ed.): Museo Castro de Chao Samartín. Catálogo. Consejería de Cultura y Turismo del Principado de Asturias y Asociación de Amigos del Parque Histórico del Navia. Oviedo, 61-67.

García y Bellido, A. (1941): “El castro de Coaña (Asturias) y algunas notas sobre el posible origen de esta cultura)" AEspA, 42: 188-217.

García y Bellido, A. (1942): "El castro de Coaña (Asturias). Nuevas aportaciones" AEspA, 42: 216-244.

García y Bellido, A. y Uría Ríu, J. (1940): “Avance a las excavaciones del Castellón de Coaña”, Revista de la Universidad de Oviedo, 2: 105-131.

García-Bellido García de Diego, M.P. (2002): "El yacimiento de Coaña y Antonio García y Bellido", en M.A. de Blas y A. Villa: Los poblados fortificados del noroeste de la Península Ibérica: formación y desarrollo de la Cultura Castreña, Navia: 39-45.

ICCROM (1984): La conservación en excavaciones arqueológicas. Roma.

Jurion-De Waha, F. (1999): "Belgique (Région bruxelloise)", Rapport sur la situation de l'Archéologie Urbaine en Europe, Council of Europe Publishing. 1999, Strasbourg, 31-39; citado en J. A. Quirós Castillo (2005): “¿Excavar en las ciudades o historiar las ciudades? El debate sobre la Arqueología Urbana a la luz de algunas experiencias europeas", Arqueología y Territorio Medieval vol. 12.1. Jaén, 107-132.

López, J. y Graña, A. (1993): "El Parque Etnográfico de Somiedo (Asturias)”, en Seminario de Parques Arqueológicos. Ministerio de Cultura. Madrid, 165-172.

Menéndez Granda, A. y Villa Valdés, A. (2009): “Os Castros de Taramundi: reseña sobre el plan director e informe relativo al avance de las excavaciones arqueológicas", Excavaciones Arqueológicas en Asturias 6, 2003-2006. Principado de Asturias. Oviedo, 455-463.

Montes López, R. y Villa Valdés, Á. (2018): “Traslado y depósito conjunto de la colección arqueológica procedente de las excavaciones del Navia-Eo a las instalaciones del centro de interpretación del Chao Samartín (Grandas de Salime)”, Excavaciones Arqueológicas en Asturias 8, 2013-2017. Oviedo, 227-234.

Ruiz de Arbulo, J. y Mar, R. (1997): “Arqueologia i planificació urbana a Tarragona. Tradició historiográfica i realitat actual”, Viure les ciutats històriques. Seminari. Recuperar la memoria urbana. L'Arqueologia en la rehabilitacio de les ciutats historiques. Documents d'Arqueologia Clássica 2. Tarragona,131-157.

Querol, Ma. A. (1993): "Filosofía y concepto de parque arqueológico", en Seminario de Parques Arqueológicos. Ministerio de Cultura. Madrid, 11-22.

Uría Ríu, J. y García y Bellido, A. (1943): “El castro de Coaña: reconstrucción gráfica de una aldea prehistórica del NW de España”, Investigación y Progreso, XIV: 65-74.

Villa Valdés, A. (1999): "Plan Arqueológico Director de la Cuenca del Navia", en Excavaciones Arqueológicas en Asturias 4. Oviedo, 205-211.

Villa Valdés, A. (2007): “Trabajos de conservación y consolidación en el poblado fortificado de Os Castros, Taramundi”, en J. Fernández Reyero y P. León Gasalla (Coord.): Intervenciones en el patrimonio cultural asturiano. Oviedo, 548-551.

Villa Valdés, A. (2009): "Sobre el Plan Arqueológico del Navia-Eo, el Parque Histórico del Navia y la creación del Museo Castro de Chao Samartín", en Museo Castro de Chao Samartín. Catálogo. Consejería de Cultura y Turismo del Principado de Asturias y Asociación de Amigos del Parque Histórico del Navia. Oviedo, 33-37. 
Villa Valdés, A. (2014): "Rescate urgente y restauración de pinturas murales en la domus del castro de Chao Samartín”, en P. León (Coord.): Intervenciones en el patrimonio cultural asturiano. Oviedo, 468-469.

Villa Valdés, A. (2019): “Apuntes sobre la Edad del Hierro en Asturias. A propósito de las excavaciones arqueológicas de García y Bellido en Coaña”, en Á. Villa Valdés \& F. Rodríguez del Cueto (Dir. \& Coord.): Arqueología castreña en Asturias. Contribuciones a la conmemoración del Día García y Bellido. Oviedo, 121-141.

Villa Valdés, A.; Gago Muñiz, O. \& Montes López, R. (2018): "Rescate urgente y consolidación de paneles pictóricos de época romana en el yacimiento arqueológico castro de Chao Samartín (Grandas de Salime)", Excavaciones Arqueológicas en Asturias 8, 2013-2017. Oviedo, 219-226.

Villa Valdés, A. \& Hevia González, S. (2014): "El Museo Castro de Chao Samartín. Justificación, contexto y desarrollo de un proyecto museológico en Grandas de Salime". Intervenciones en el Patrimonio Cultural Asturiano 2007-2014. Consejería de Educación, Cultura y Deporte del Principado de Asturias. Oviedo, 120-124.

Villa Valdés, A.; Menéndez Granda, Alv. \& Fanjul Mosteirín, J.A. (2007): “ Excavaciones arqueológicas en el poblado fortificado de Os Castros, en Taramundi”, en Excavaciones Arqueológicas en Asturias 1999-2002. Oviedo, 267-276. 
